\title{
The Drosophila ortholog of TMEM18 regulates insulin and glucagon-like signaling
}

\author{
Lyle Wiemerslage, Priya A Gohel, Giulia Maestri, Torfi G Hilmarsson, \\ Michel Mickael, Robert Fredriksson, Michael J Williams and Helgi B Schiöth
}

Department of Neuroscience, Functional Pharmacology, Uppsala University, Uppsala, Sweden

Correspondence

should be addressed

to L Wiemerslage

Email

lyle.wiemerslage@neuro.uu.se

\begin{abstract}
Transmembrane protein 18 (TMEM18) is an ill-described, obesity-related gene, but few studies have explored its molecular function. We found single-nucleotide polymorphism data, suggesting that TMEM18 may be involved in the regulation/physiology of

metabolic syndrome based on associations with insulin, homeostatic model assessment- $\beta$ (HOMA $\beta)$, triglycerides, and blood sugar. We then found an ortholog in the Drosophila genome, knocked down Drosophila Tmem18 specifically in insulin-producing cells, and tested for its effects on metabolic function. Our results suggest that TMEM18 affects substrate levels through insulin and glucagon signaling, and its downregulation induces a metabolic state resembling type 2 diabetes. This work is the first to experimentally describe the metabolic consequences of TMEM18 knockdown, and further supports its

association with obesity.
\end{abstract}

Key Words

- Drosophila

- fly

- insulin

- obesity

- SNP

- TMEM18

- CG30051

- glucagon

- $\mathrm{AKH}$

Journal of Endocrinology

(2016) 229, 233-243

\section{Introduction}

Obesity and its sequelae (diabetes, heart disease, cancer, etc.) arguably make it the most important health concern in modern time (Calle et al. 2003, Haslam \& James 2005, Barness et al. 2007), and the molecular mechanisms of obesity, including the genes involved, must be investigated. Our laboratory has previously described orthologs of Drosophila melanogaster from 22 human genes related to obesity (Williams et al. 2012). Flies are an excellent model system for studying a variety of molecular pathways, as well as behavior. Specifically for obesity, they have organs that function similar to the human pancreas with corresponding orthologs of insulin and glucagon: insulin-like protein 2 (Ilp2) and adipokinetic hormone $(\mathrm{AKH})$, respectively (Kim \& Rulifson 2004,
Nassel et al. 2013, Cao et al. 2014). Moreover, the suite of genetic tools available in Drosophila allows for highly specific experimentation. Thus, flies are a valuable model when exploring the function of ill-described genes. We investigate the metabolic effects of transmembrane protein 18 (Tmem18), the Drosophila ortholog of human transmembrane protein 18 (TMEM18).

Few studies have explored the function of TMEM18. Genome-wide association studies (GWAS) have identified risk alleles in/near TMEM18, associated with body mass index (BMI) (Thorleifsson et al. 2009, Willer et al. 2009) and type 2 diabetes (Kalnina et al. 2013). TMEM18 is ubiquitously expressed throughout the body and central nervous system-including the hypothalamus and other

Published by Bioscientifica Ltd 
brain areas important for metabolism (Willer et al. 2009, Almen et al. 2010, Rask-Andersen et al. 2012), and has decreased expression in subcutaneous adipose tissue compared with visceral adipose tissue (Rohde et al. 2014). In human cell lines, TMEM18 was found in the nuclear membrane and was able to bind DNA (Jurvansuu \& Goldman 2011), although this DNA-binding element appears to be missing in fly (Almen et al. 2010). Lastly, an in vitro study in mouse demonstrated that TMEM18 is involved in neuronal cell migration (Jurvansuu et al. 2008). To date, how TMEM18 affects metabolism remains unknown. Using publically available human data and the genetically tractable Drosophila model system, we provide the first molecular description of how TMEM18 may be involved in obesity and type 2 diabetes.

\section{Materials and methods}

\section{Single-nucleotide polymorphism analysis}

A list of 316 human single-nucleotide polymorphisms (SNPs) for TMEM18 was downloaded from the National Center for Biotechnology Information dbSNP database on September 5, 2014. This list was then compared against the results from all available datasets (also downloaded on 5 September 2014) from the following databases: GIANT (http://www.broadinstitute.org/collaboration/ giant/), MAGIC (www.magicinvestigators.org), DIAGRAM (http://diagram-consortium.org/), GLGC (http://www. sph.umich.edu/csg/abecasis/public/lipids2010/), and CARDIoGRAM (http://www.cardiogramplusc4d.org/). Each database has carried out analysis for significance and made corrections for multiple testing. We only report SNPs with a $P$-value $<0.05$ and individual studies are cited with their corresponding data in the results. Data from the ENCODE project (Rosenbloom et al. 2013) was visualized in the UCSC Genome Browser (http://genome.ucsc.edu/) using the Human February 2009 (GRCh37/hg19) Assembly (Rosenbloom et al. 2012).

\section{Phylogenetic analysis}

Orthologous protein and genomic sequences of TMEM18 were used to construct a multiple sequence alignment and evolutionary tree as well as to test selective pressure via phylogenetic analysis by maximum likelihood (PAMIL). A full description is available in the Supplementary Materials and methods (see section on supplementary data given at the end of this article).

\section{Fly stocks and maintenance}

Our phylogenetic analysis indicates that CG30051 is a Drosophila ortholog of human TMEM18; therefore, we have renamed the gene Tmem18. $w^{1118}$; $\mathrm{P}\{\mathrm{v} 43067\}$ flies containing Tmem18-RNAi were obtained from the Vienna Drosophila RNAi Centre (Vienna, Austria). w; Ilp2-GAL4 was a gift from Dr Eric Rulifson (University of California, San Francisco, CA, USA) (Wang et al. 2007). $t G P H$ flies were obtained from Bloomington Stock Center (Indiana University, Bloomington, IN, USA). Ilp2-GAL4;tGPH flies were bred into a homozygous strain by our laboratory. All flies, unless otherwise stated, were maintained on enriched Jazz mix standard fly food (Thermo Fisher Scientific). Flies were maintained at $25^{\circ} \mathrm{C}$ in an incubator at $60 \%$ humidity on a $12 \mathrm{~h}$ light: $12 \mathrm{~h}$ darkness cycle. Flies crossed with GAL4 drivers and controls were raised at $18^{\circ} \mathrm{C}$ until the adults emerged; once collected, adults were raised $5-7$ days at $29^{\circ} \mathrm{C}$. In all assays, the GAL4 drivers and UAS transgenic flies were crossed to $w^{1118}$ flies and their F1 progeny were used as controls.

\section{Capillary feeding assay}

This method was modified from the original (Ja et al. 2007) and performed as previously described (Williams et al. 2014b).

\section{Trehalose, glucose, and lipid analysis}

Circulating and stored carbohydrate levels were measured via a modified method from the original (Tennessen et al. 2011) (see Supplementary Materials and methods). Lipid measurements were performed as described previously (Williams et al. 2014b).

\section{Glucose stimulus assay}

To investigate InR/PI3K signaling activity, we utilized a previously described method using $t G P H$ flies (Britton et al. 2002) (Supplementary Materials and methods).

\section{RNA purification, cDNA synthesis, and quantitative RT-PCR}

RNA purification, cDNA synthesis, and quantitative RT-PCR (qRT-PCR) was performed as described previously (Williams et al. 2014a). Samples were prepared from either 15 flies or 30 fly heads (depending on experiment), and cDNA synthesis was confirmed by PCR followed by agarose

Published by Bioscientifica Ltd. 
gel electrophoresis. Relative expression levels of two housekeeping genes (Rp49 and RpL11) and of the genes of interest were determined with qRT-PCR. The following primers were used: Tmem18 F: 5'-GTTTGTTTTGACTCGGCTTTC-3', R: 5'-CCATTGATCTCGTTGACCTC-3'; Dubai F: 5'- CAAGCAGTTCAAGTACGAT-3', R: 5'-TTACTGTGACGCTCTCAT-3'; Rpl11 F: 5'-CCATCGGTATCTATGGTCTGGA-3'， R: 5'-CATCGTATTTCTGCTGGAACCA-3'; Rp49 F: 5'-CACACCAAATCTTACAAAATGTGTGA-3', R: 5'-AATCCGGCCTTGCACATG-3'; Ilp2 F: 5'-TCTGCAGTGAAAAGCTCAACGA-3', R: 5'-TCGGCACCGGGCATG-3'; Ilp3 F: 5'-TGAACCGAACTATCACTCAACAGTCT-3', R: 5'-AGAGAACTTTGGACCCCGTGAA-3'; Ilp5 F: 5'-GAGGCACCTTGGGCCTATTC-3'， R: 5'-CATGTGGTGAGATTCGGAGCTA-3'; Akh F: 5'-CTGGTCCTGGAACCTTTT-3', R: 5'-GAGCTGTGCCTGAGATTG-3'; AkhR 5'-TCCGTAGCAGTAGATGAA-3', R: 5'-AGGAGCGACTTTGATGAG-3'.

\section{Statistical analysis}

Before analysis, all distributions were tested for normality and homogeneity of variance. For experiments with multiple comparisons, differences between groups were first tested with an ANOVA followed by pairwise $t$-test comparisons with the Tukey's honestly significant difference correction. Data are reported as mean \pm 1 s.E.M.

\section{Results}

\section{TMEM18 appears to regulate metabolic syndrome via effects on insulin}

To find clues for TMEM18's relationship with obesity, we searched the GIANT, MAGIC, DIAGRAM, GLGC, and CARDIoGRAM databases for SNPs linked with metabolic phenotypes. Of 316 SNPs for TMEM18, we found 13 significant for at least one metabolic phenotype: 12 with obesity (Speliotes et al. 2010), 9 with fasting insulin levels (Manning et al. 2012), 6 with the homeostatic model assessment- $\beta$ (HOMA $\beta$ ) (Dupuis et al. 2010), 5 with triglycerides (Teslovich et al. 2010), 4 with BMI (Speliotes et al. 2010), and 1 with HbA1c (Soranzo et al. 2010) (Table 1). Many of these SNPs were significant for multiple phenotypes (Fig. 1). Control experiments show that these results are not likely due to excessive food intake or diet (see Supplementary Materials and methods, and Supplementary Figs 2 and 3). Furthermore, these alleles with multiple phenotypes had effects in the same direction, that is, for an unhealthy metabolic phenotype. For example, the risk allele for rs10164967 was associated with increased insulin levels, increased НОМА $\beta$, and increased triglyceride levels (Table 1). These findings suggest that TMEM18 affects obesity through insulin signaling and/or regulation of substrate levels, and thus is likely an important gene for obesity, metabolic syndrome, and type 2 diabetes.

Next, we determined if the 13 SNPs mentioned previously appeared in important regulatory areas. The online tool RegulomeDB reported two SNPs that likely affect the binding of regulatory elements or expression of TMEM18: rs17042334, which is associated with increased insulin, НОMA $\beta$, and triglyceride levels, and rs17729501, which is associated with obesity (Boyle et al. 2012). Moreover, we found that both of these SNPs reside in the 3' UTR region of TMEM18 and, using data from the ENCODE project, are within $1 \mathrm{~kb}$ of an insulator (and DNase activity), which overlaps with transcription factor binding sites for CTCF, Fos, and Jun (Rosenbloom et al. 2012, 2013). In addition, the online tool mrSNP found 11 miRNAs whose binding may be affected by 3 of the 13 SNPs described in our analysis: rs17042334, rs17729501, and rs3187671 (notably, these include the two SNPs identified by RegulomeDB) (for an improved phylogenic analysis see the Supplementary Materials and methods, Supplementary Figure 1, and Supplementary Tables 1 and 2 (see section on supplementary data given at the end of this article)) (Deveci et al. 2014). Thus, these SNPs may be important biomarkers, and TMEM18 could be regulated by growth signaling.

\section{The D. melanogaster TMEM18 ortholog has a comparable pattern of expression between humans and flies}

CG30051 is the putative ortholog of TMEM18 in the fly D. melanogaster. A basic local alignment search tool for protein (BLASTp) (Altschul et al. 1990) search using the human TMEM18 protein sequence as a query (NP_690047.21) reported CG30051 as the only significant hit in D. melanogaster ( $e$-value $\left.=1^{-28}\right)$, and previous work found high conservation in the transmembrane domains of TMEM18 across several species, including D. melanogaster (Almen et al. 2010). We further support these findings with an improved phylogenic analysis (see the Supplementary Materials and methods). Then we compared absolute, tissue-specific expression data of TMEM18 from the Human eFP Browser with analogous

Published by Bioscientifica Ltd 
Table 1 TMEM18 SNPs associated with multiple metabolic phenotypes.

\begin{tabular}{|c|c|c|c|c|c|c|}
\hline Phenotype & rs ID & Major allele & Minor allele & $P$-value & Effect & $\begin{array}{c}\text { Minor allele } \\
\text { frequency (\%) }\end{array}$ \\
\hline \multirow[t]{12}{*}{ Obesity } & rs12714416 & a & g & 0.006 & NA & 17 \\
\hline & rs12990777 & $g$ & $\mathrm{a}$ & 0.006 & & 17 \\
\hline & rs2293083 & c & $g$ & 0.005 & & 17 \\
\hline & rs2293084 & $g$ & $\mathrm{t}$ & 0.017 & & 61 \\
\hline & rs2293082 & $g$ & c & 0.039 & & 8 \\
\hline & rs10172821 & c & $\mathrm{t}$ & 0.024 & & 9 \\
\hline & rs17729501 & c & $\mathrm{t}$ & 0.013 & & 9 \\
\hline & rs3187671 & $\mathrm{t}$ & c & 0.024 & & 9 \\
\hline & rs10164967 & c & g & 0.038 & & 8 \\
\hline & rs10168696 & c & $\mathrm{t}$ & 0.038 & & 5 \\
\hline & rs13002433 & c & $\mathrm{t}$ & 0.038 & & 8 \\
\hline & rs4241323 & a & $g$ & 0.038 & & 8 \\
\hline \multirow{9}{*}{$\begin{array}{l}\text { Insulin levels } \\
\text { (fasting) }\end{array}$} & rs10164967 & c & g & 0.012 & 0.012 & 8 \\
\hline & rs10168696 & $\mathrm{t}$ & c & 0.012 & -0.012 & 5 \\
\hline & rs12714416 & a & g & 0.017 & 0.009 & 17 \\
\hline & rs12990777 & $a$ & g & 0.015 & -0.009 & 19 \\
\hline & rs13002433 & $\mathrm{t}$ & c & 0.012 & -0.012 & 8 \\
\hline & rs17042334 & $\mathrm{t}$ & c & 0.013 & 0.012 & 9 \\
\hline & rs2293082 & c & g & 0.013 & -0.012 & 8 \\
\hline & rs2293083 & c & g & 0.015 & 0.009 & 17 \\
\hline & rs4241323 & $\mathrm{a}$ & $g$ & 0.012 & 0.012 & 8 \\
\hline \multirow[t]{6}{*}{ HOMAb } & rs10164967 & c & $g$ & 0.008 & 0.013 & 8 \\
\hline & rs10168696 & $\mathrm{t}$ & c & 0.007 & -0.013 & 5 \\
\hline & rs13002433 & $\mathrm{t}$ & c & 0.007 & -0.013 & 8 \\
\hline & rs17042334 & $\mathrm{t}$ & c & 0.010 & 0.013 & 9 \\
\hline & rs2293082 & c & g & 0.008 & -0.013 & 8 \\
\hline & rs4241323 & a & g & 0.007 & 0.013 & 8 \\
\hline \multirow[t]{5}{*}{ Triglycerides } & rs10164967 & c & g & 0.034 & 0.011 & NR \\
\hline & rs10168696 & $\mathrm{t}$ & c & 0.037 & -0.011 & \\
\hline & rs13002433 & $\mathrm{t}$ & c & 0.041 & -0.011 & \\
\hline & rs17042334 & $\mathrm{t}$ & c & 0.044 & 0.011 & \\
\hline & rs4241323 & $\mathrm{a}$ & $g$ & 0.040 & 0.011 & \\
\hline \multirow[t]{4}{*}{ BMI } & rs12714416 & a & g & 0.023 & NR & 17 \\
\hline & rs12990777 & $\mathrm{g}$ & a & 0.022 & & 17 \\
\hline & rs2293083 & c & $g$ & 0.023 & & 17 \\
\hline & rs2293084 & g & $\mathrm{t}$ & 0.017 & & 61 \\
\hline $\mathrm{Hb} 1 \mathrm{Ac}$ & rs2293084 & $\mathrm{t}$ & $\mathrm{g}$ & 0.015 & -0.009 & 43 \\
\hline
\end{tabular}

List of significant SNPs for metabolic phenotypes. An initial list of 316 putative human SNPs for TMEM18 was downloaded from the NCBI dbSNP database and matched with significant SNPs from the following GWAS databases: GIANT, MAGIC, DIAGRAM, GLGC, and CARDIoGRAM. Each database has tested for significance and made corrections for multiple testing. Only SNPs with a $P$-value $<0.05$ are reported. NA, not applicable; NR, not reported.

data of CG30051 from FlyAtlas 2. For both genes, there was high expression in the ovaries, testes, and salivary glands. Other tissues showed even, ubiquitous expression (Winter et al. 2007, Robinson et al. 2013). Data from the Human Protein Atlas further supported this comparison, showing high expression not only for the same areas but also for pancreas and stomach (Uhlén et al. 2010, 2015). CG30051 is likely an ortholog of TMEM18; thus, we renamed the Drosophila gene Tmem18.

\section{Tmem18 knockdown in insulin-producing cells has effects on starvation}

As Tmem18 was associated with insulin in the human data, we wanted to limit its manipulation to the insulinproducing cells (IPCs) in Drosophila and test for an effect from RNAi knockdown specifically in these cells (for knockdown of Tmem18, see the Supplementary Materials and methods). Ilps are produced by 14 IPCs

Published by Bioscientifica Ltd. 


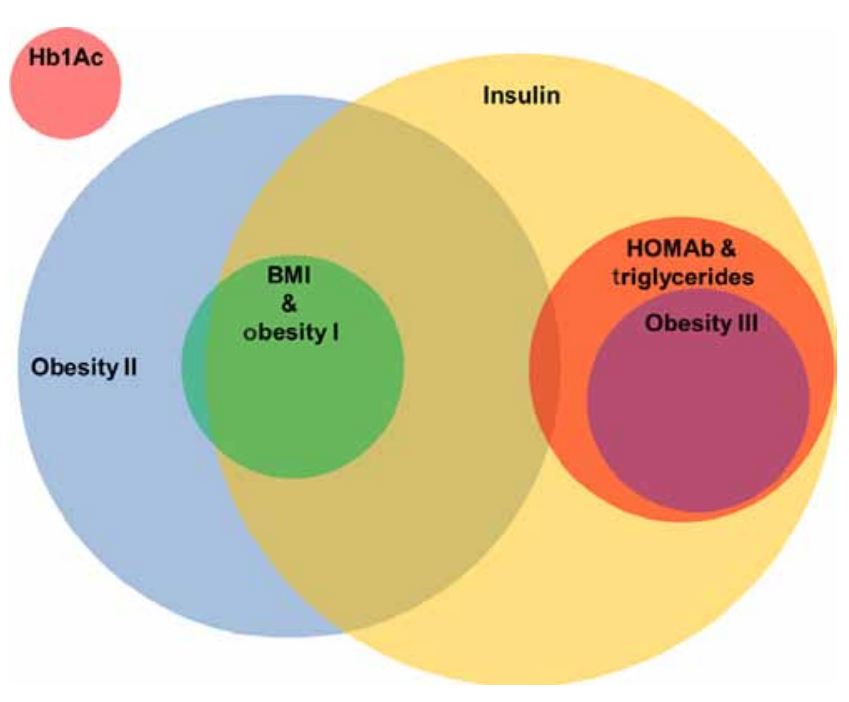

Figure 1

Many SNPs for TMEM18 are associated with multiple metabolic phenotypes. Venn diagram representing the overlap of SNPs for a given phenotype. SNPs associated with insulin levels were the most encompassing, whereas the SNP associated with the blood glucose test, $\mathrm{Hb} 1 \mathrm{Ac}$, did not overlap with any other phenotypes, as did some SNPs associated with class II obesity, and 1 with BMI and class I obesity. Obesity was broken up into three groups based on BMI: class I (BMI $\left.=30-34.99 \mathrm{~kg} / \mathrm{m}^{2}\right)$, class II (BMI $\left.=35-39.99 \mathrm{~kg} / \mathrm{m}^{2}\right)$, and class III $\left(B M I>40 \mathrm{~kg} / \mathrm{m}^{2}\right)$. Interestingly, SNPs associated with the super obese (class III) overlapped completely with the HOMAb and triglyceride levels, which were also completely contained within insulin levels. Also, overlapping SNPs always had effects in the same direction, for example, the risk allele for rs 10164967 was associated with increased insulin levels, HOMAb, and triglyceride levels. Thus, SNPs that overlap for multiple phenotypes may be important biomarkers. A full colour version of this figure is available at http://dx.doi.org/10.1530/JOE-16-0040.

with physiological function similar to mammalian pancreatic beta cells (Nassel et al. 2013). Moreover, RNAseq experiments from laser-dissected IPCs in Drosophila larvae identified Tmem18 expression in those cells (Cao et al. 2014). To limit Tmem18-RNAi specifically to IPCs, we drove expression with an Ilp2 (Ilp2-GAL4) construct.

First, we performed a starvation assay using UAS-Tmem18-RNAi flies crossed with Ilp2-GAL4 flies (Ilp2>Tmem18-RNAi). Adult flies, aged 5-7 days, were starved until death. The Ilp2>Tmem18-RNAi flies were $26 \%$ less resistant to starvation compared with controls (Fig. 2A). Next, we found that the level of Tmem18 expression changes in control flies during starvation. The control flies were from a cross between Canton-S and Oregon-R-C (named CSORC). The expression of Tmem 18 decreased $79 \%$ by $12 \mathrm{~h}$ starvation (Fig. $2 \mathrm{~B}$ ). Thus, starvation affects the expression of Tmem18, and reduced the expression of Tmem18 in the brain, which likely has negative consequences on metabolic functions.
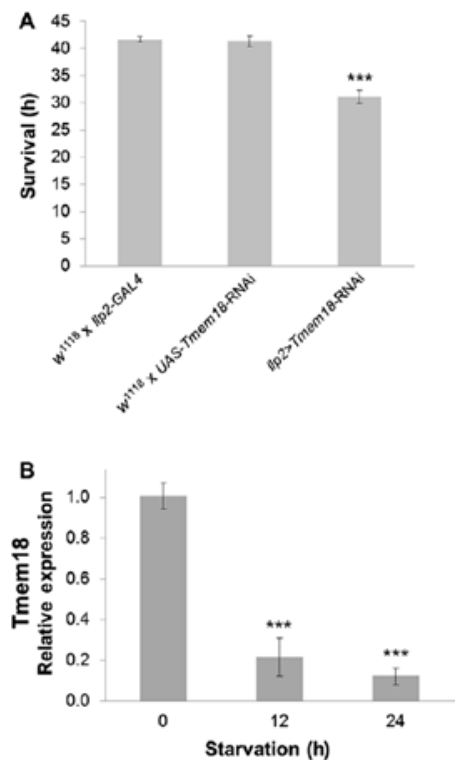

Figure 2

CG30051 is involved during starvation. (A) Flies lacking CG30051 in the IPCs were less resistant to starvation compared to two control crosses. CG30051 was knocked down with RNAi specifically in the IPCs using the IIp2-GAL4 driver. Adult, male flies, aged 5-7 days post-eclosion, were starved until death in vials containing $1 \%$ agarose $(n=32$ flies for each strain). (B) CG30051 expression decreased during starvation. The heads of adult, male flies, aged 5-7 days post-eclosion from the CSORC strain, were collected for qPCR analysis after 0,12 , or $24 \mathrm{~h}$ during starvation. CG30051 expression decreased by $12 \mathrm{~h}$ and remained decreased at $24 \mathrm{~h}$ ( $n=$ number of samples, three replicates per sample; $n=5$ for all time points; Tukey's HSD test, $* * * P<0.001$ ).

\section{Knockdown of CG30051 in IPCs changes metabolic profile}

We next measured the carbohydrate and lipid levels of Ilp2 $>$ Tmem18-RNAi flies. Four different measurements are made for carbohydrate levels: body stores of glycogen and trehalose, and circulating levels of glucose and trehalose in hemolymph. All five substrates were measured in flies fed ad libitum, as well as after 12 and $24 \mathrm{~h}$ starvation. We found increases in all metabolic measurements when Tmem18 was knocked down specifically in the IPCs.

For stored carbohydrates, glycogen and trehalose levels in body were increased 14 and 30\%, respectively, when fed ad libitum, and elevations remained after 12 and $24 \mathrm{~h}$ starvation (Fig. 3A and B). For circulating carbohydrate stores, glucose and trehalose levels in the hemolymph increased 86 and 60\%, respectively, but were not similarly maintained throughout starvation (Fig. 3C and D). Lastly, the total lipid content also increased $29 \%$ in flies fed ad libitum, but not significantly during starvation (Fig. 3E). Thus, Tmem18 knockdown increases basal substrate levels, which are partially maintained

Published by Bioscientifica Ltd. 
A

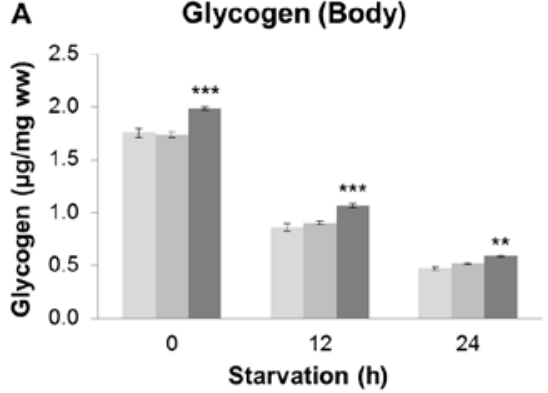

C

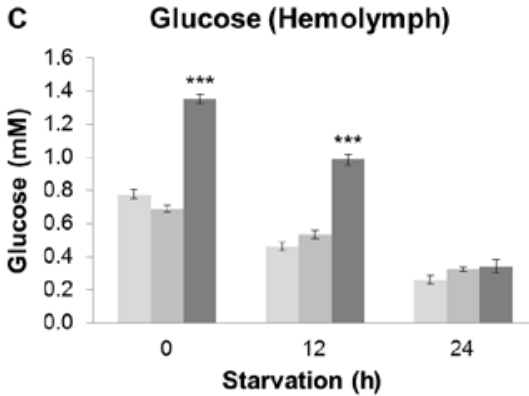

E

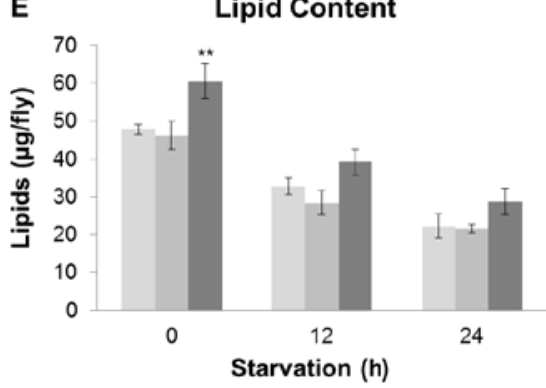

B

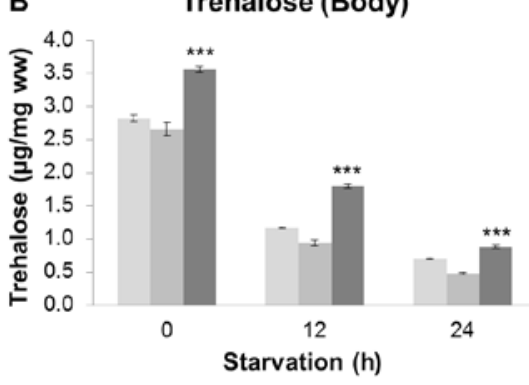

D

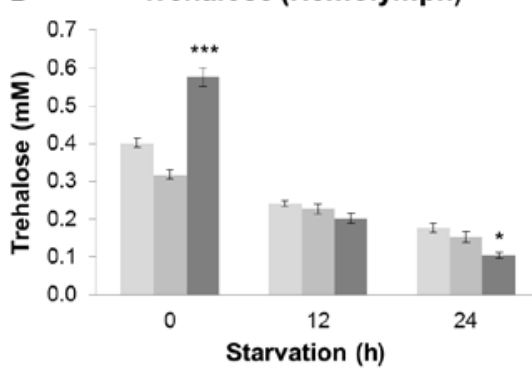

$w^{1118} \times$ IIP2-GAL4

$w^{1118} \times$ UAS-Tmem18-RNAi

IIp2>TMEm18-RNAi

\begin{abstract}
Figure 3
Knockdown of CG30051 in IPCs changes the metabolic profile of the fly. Both circulating and body levels of carbohydrates, as well as total lipid content, were increased. CG30051 was knocked down specifically in the IPCs and compared to two control crosses. Adult, male flies, aged 5-7 days post-eclosion, were collected for analysis at 0,12 , or $24 \mathrm{~h}$ starvation ( $n=$ number of samples; $n=8$ for all strains at each time point for all experiments except for lipids; Tukey's HSD test, ${ }^{*} P<0.05$, $* * * P<0.001)$. (A) Glycogen stores were increased at 0,12 , and $24 \mathrm{~h}$. (B) Body trehalose levels were also increased at 0, 12, and $24 \mathrm{~h}$. (C) Circulating glucose in the hemolymph was increased at 0 and $12 \mathrm{~h}$. (D) Circulating trehalose in the hemolymph was increased at $0 \mathrm{~h}$ but decreased at $24 \mathrm{~h}$. (E) Lipid content per fly increased at $0 \mathrm{~h}$ ( $n=$ number of samples; Ilp2-GAL4 $\times W^{1118:} 0 \mathrm{~h}, n=7,12 \mathrm{~h}, n=8$, $24 \mathrm{~h}, n=6 ; w^{1118} \times C G 30051$-RNAi: $0 \mathrm{~h}, n=8,12 \mathrm{~h}$, $n=7,24$ h, $n=5$; Ilp2-GAL4>CG30051-RNAi: 0 h, $n=7,12 \mathrm{~h}, n=6,24 \mathrm{~h}, n=6$; Tukey's HSD test, $* * P<0.01, * * * P<0.001)$.
\end{abstract}

throughout starvation. Control experiments show that these results are not likely due to excessive food intake or diet (see the Supplementary Materials and methods).

\section{Evidence for the modulation of metabolic genes by TMEM18}

We next determined if transcript levels of metabolic proteins were affected by Tmem18 knockdown in the same population of cells. qRT-PCR experiments tested a subset of the insulin orthologs Ilp2, Ilp3, and Ilp5, as well as the Drosophila insulin receptor $(\operatorname{In} R)$. In addition, we tested adipokinetic hormone $(A k h)$, which functions as glucagon in fly (Kim \& Rulifson 2004, Buch et al. 2008), and its receptor $A k h R$. Expression of $I l p 2$ and $I n R$, and $A k h$ decreased $\sim 33, \sim 42$, and $\sim 59 \%$, respectively, compared with controls, whereas increases were found in the Ilp3 and Ilp5 transcripts: $\sim 43$ and $\sim 139 \%$, respectively, compared with control levels. AkhR was the only transcript unchanged (Fig. 4). Thus, Tmem 18 could be affecting metabolism via regulation of metabolic proteins.
Tmem18 knockdown inhibits the response to feeding in larval fat body cells

Previous work in Drosophila established a method to visualize insulin signaling in the fat body cells of larvae using Ilp2-GAL4; $t G P H$ transgenic flies (Britton et al. 2002). Briefly, a $\beta$-tubulin construct drives the expression of green fluorescent protein (GFP) tagged to the PH domain of the Drosophila general receptor for phosphoinositides-1 (GRP1). In starved conditions, the PH-GFP chimeric protein is kept perinuclear but is trafficked to the cell membrane after feeding, that is, during insulin signaling. To determine if Tmem18 is involved in the cellular response to insulin signaling, we crossed Ilp2-GAL4; tGPH flies with either UAS-Tmem18RNAi or $w^{1118}$ control flies. Second instar larvae from each cross were collected and starved for $30 \mathrm{~min}$ and then refed for $30 \mathrm{~min}$ after starvation. The fat bodies were dissected from larvae in both the starved and fed states, and visualized via the GFP signal. In both crosses, the GFP signal was perinuclear during starvation, showing

Published by Bioscientifica Ltd. 


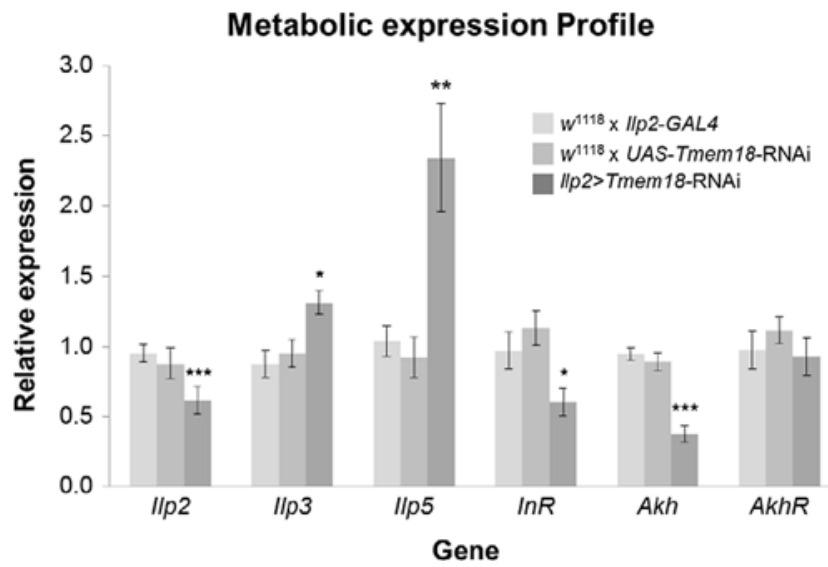

Figure 4

Knockdown of CG30051 in IPCs changes the expression profile of metabolic genes. Whole, male flies, aged 5-7 days post-eclosion, were collected and processed for qPCR. Primers for six metabolic genes (IIp2, $\| l p 3, I / p 5, I n R, A k h$, and $A k h R$ ) were tested in the IIp2 > CG30051-RNAi cross versus two different control crosses. Expression in the I/p2 > CG30051-RNAi cross was decreased in I/p2, InR, and Akh; increased in IIp3 and IIp5; and unchanged for $A k h R$ ( $n=$ number of samples, three replicates per sample; $n=8$ for all groups; Tukey's HSD test $n,{ }^{*} P<0.05$, $* * P<0.01, * * * P<0.001)$.

ill-defined borders (Fig. 5A and B). During the refed state, however, the GFP signal in the control cross $\left(w^{1118} \times\right.$ Ilp2$G A L 4 ; t G P H)$ saturated the cell membrane demarcating cell borders (Fig. 5C, white arrows), whereas in the Ilp2GAL4; $t G P H \times U A S$-Tmem18-RNAi cross, the GFP signal remained perinuclear (Fig. 5D). Thus, Tmem18 is likely important for proper cellular responses to insulin or other signal transductions involving phosphoinositides.

\section{Discussion}

In this work, we examine the possible metabolic function of the obesity-related gene TMEM18. We find human SNP data, suggesting that TMEM18 may affect obesity via insulin signaling. Based on these clues from the human data, we test the Drosophila ortholog of TMEM18 (CG30051), hence renamed Tmem18, for effects on metabolic function. Using an RNAi construct with the GAL4-UAS system, we knock down Tmem18 expression specifically in a cell population that functions similar to human pancreatic beta cells. We find increased levels of circulating and stored carbohydrates, as well as increased lipid levels. We also find different expression levels of metabolic proteins orthologous to insulin and glucagon. Our results suggest that TMEM18 may affect substrate levels by disrupting insulin and glucagon signaling. This work is the first to experimentally describe the metabolic consequences of TMEM18, and further supports its association with obesity.

TMEM18 was initially identified by GWAS for a relationship with BMI (Thorleifsson et al. 2009,

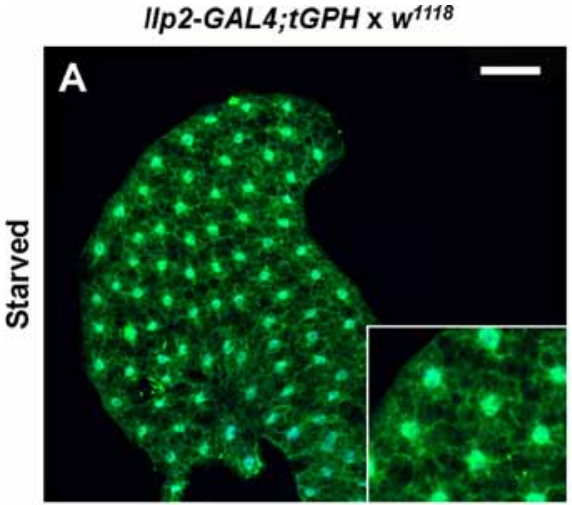

IIp2-GAL4;TGPH x UAS-Tmem18-RNAi

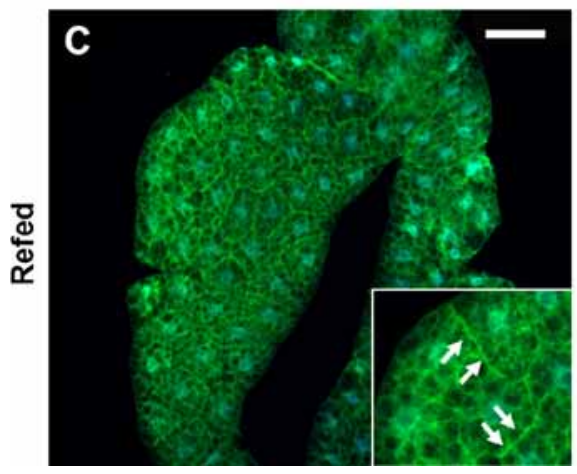

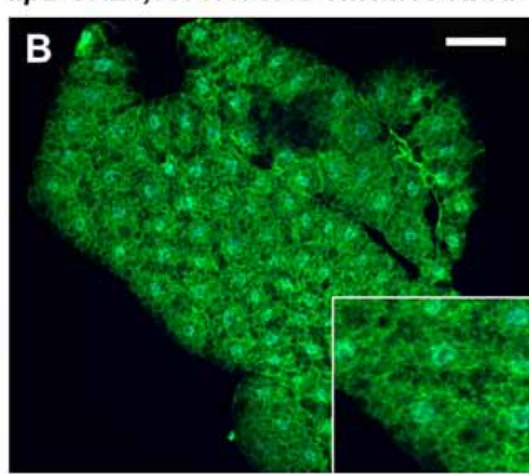

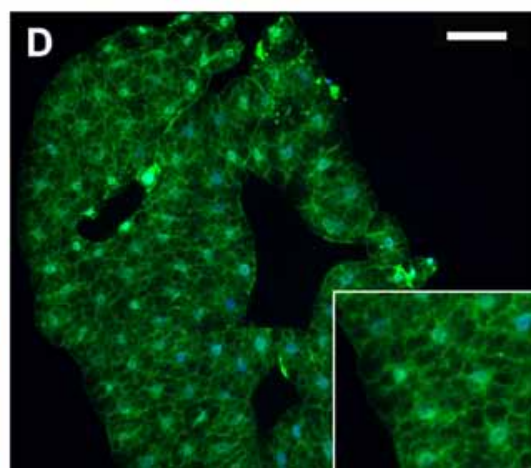

\section{Figure 5}

Insulin signaling is disrupted in fat body cells lacking CG30051. Fat body cells from the third instar larvae were dissected from IIp2-GAL4; $t G P H$ flies crossed to either $w^{1118}$ or UAS-CG30051-RNAi flies and stained via immunocytochemistry (nuclei shown in blue). Ilp2-GAL4; $t G P H$ flies express GFP tagged to GRP1 protein, which is trafficked to the membrane from its perinuclear locale during phosphoinositide signaling cascades, for example, during insulin signaling in response to feeding. (A) Fat body cells starved for $30 \mathrm{~min}$ in the IIp2-GAL4; tGPH $\times$ W $^{1118}$ control cross. The GFP signal is mostly perinuclear, indicating an absence of insulin signaling. (B) Starved cells in the IIp2-GAL4; tGPH $\times$ UAS-CG30051-RNAi cross. (C) Fat body cells refed for $30 \mathrm{~min}$ after starvation in the Ilp2-GAL4; $t G P H \times w^{1118}$ control cross. The GFP signal is now largely trafficked to the cell membrane, indicating a response to insulin signaling. (D) Refed cells in the Ilp2-

GAL4; $T$ GPH $\times$ UAS-CG30051-RNAi cross. The GFP signal remains perinuclear, indicating reduced response to insulin signaling. Images taken at $100 \times$ magnification (scale bar $=100 \times 20 \mu \mathrm{m}$ ). Insets are from each respective image (white arrows in C show cell borders). A full colour version of this figure is available at http://dx.doi. org/10.1530/JOE-16-0040. http://joe.endocrinology-journals.org DOI: 10.1530/JOE-16-0040
(C) 2016 Society for Endocrinology Printed in Great Britain 
Willer et al. 2009). We searched several GWAS databases for TMEM18 SNPs associated with other metabolic phenotypes. Besides BMI, there were SNPs associated with obesity, insulin levels, НОМА $\beta$, triglycerides, and blood glucose. Many of these SNPs had overlapping significance for multiple phenotypes. Furthermore, two SNPs are predicted to affect the binding of regulatory factors and/or the expression of TMEM18, and three SNPs were predicted to affect the binding of 11 different miRNAs. Thus, our work reveals several SNPs (particularly rs17042334 and rs17729501) that may be interesting biomarkers.

We then extend the findings from human GWAS databases with molecular experiments using the genetically tractable $D$. melanogaster, manipulating its TMEM18 ortholog. The transmembrane sequences of Drosophila Tmem 18 have highly shared sequence identity with other TMEM18 orthologs (Almen et al. 2010). And interestingly, no paralogs of TMEM18 were found in any species studied. This might indicate that duplication of TMEM18 disrupts the normal functioning of a single copy. Indeed, our PAML analysis indicated that the gene is under strong negative selection. Thus, TMEM18 is likely an important gene with strict evolutionary pressure toward conservation. Furthermore, human TMEM18 and Tmem18 had similar tissue-specific expression patterns. From all this, Tmem18 is likely to be a good model to study the function of human TMEM18. Using an RNAi construct with expression driven by the GAL4-UAS system (Brand \& Perrimon 1993), we knocked down Tmem18 expression specifically in the IPCs of Drosophila. These cells function physiologically similar to the mammalian pancreas (Cao et al. 2014), releasing Drosophila insulinlike proteins (Ilp) (Nassel et al. 2013, Cao et al. 2014). Using this knockdown, we tested substrate levels and expression of metabolic genes.

Knockdown of Tmem18 in the IPCs changed the metabolic profile of the flies, as well as the transcriptional profile of metabolic genes. All substrate levels were increased: glycogen and trehalose stored in the body, circulating glucose and trehalose in hemolymph (orthologous to mammalian blood glucose), and total lipids. The same changes in substrate levels also happened in flies with inhibited or ablated IPCs (Rulifson et al. 2002, Broughton et al. 2005, Slack et al. 2010) and are analogous to a diabetes-like affliction (Kannan \& Fridell 2013). In this state, insulin levels are reduced and substrates are mobilized, similarly as in starvation. Indeed, Tmem18 was also reduced during starvation in control flies, and substrate levels remained elevated throughout starvation in flies lacking Tmem18

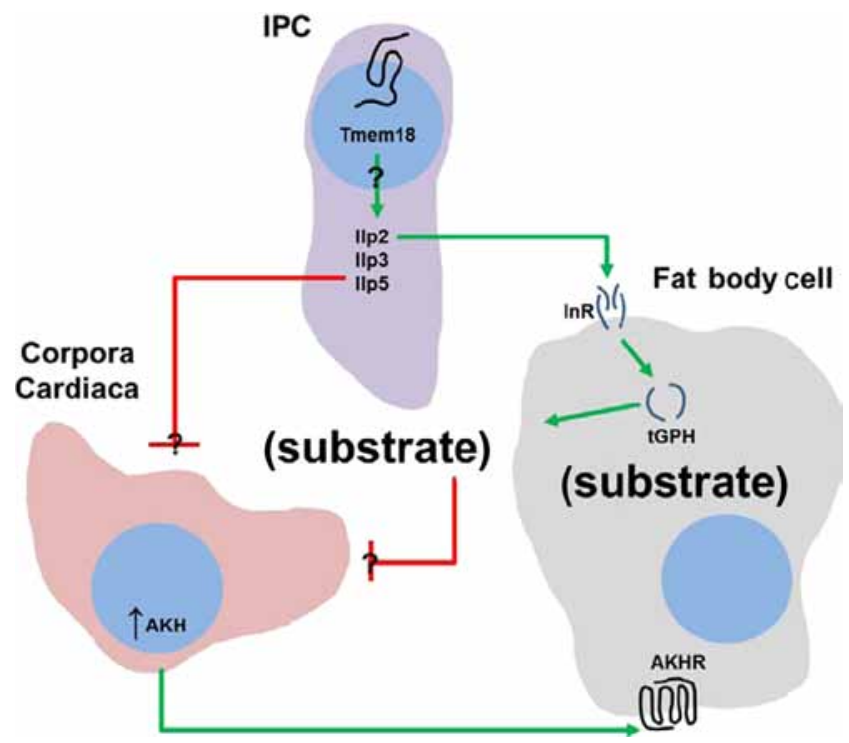

Figure 6

Theoretical mechanism of CG30051 in metabolism. CG30051 in the IPCs may exist in the nuclear membrane, where it modulates expression of Ilp proteins via an unknown mechanism. Knockdown of CG30051 resulted in decreased IIp2 but increased IIp3 and IIp5. The increased IIp3 and I/p5 may have resulted in the downregulation of $A k h$ in the corpora cardiaca cells. InR signaling was also downregulated by CG30051 knockdown and illustrated by the failure of tGPH (a GFP-tagged molecule indicating signal transduction involving phosphoinositides) to be mobilized to the cell membrane following insulin signaling. Thus, the elevated levels of substrate (glucose, trehalose, and lipids), both stored and circulating, are likely the result of an insulin-resistant phenotype induced by disrupted insulin and AKH signaling. A full colour version of this figure is available at http://dx.doi.org/10.1530/JOE-16-0040.

in IPCs. Thus, Tmem18 knockdown appears to induce a starvation-like metabolic phenotype.

During starvation, insulin protein levels decrease, but in flies lacking Tmem18 in the IPCs, we found both increases and decreases for the different Ilp transcripts. Drosophila has eight Ilp genes, but Ilp2, Ilp3, Ilp5, and Ilp6 are the best studied. All are expressed in IPCs, except Ilp6, which is expressed in the fat body with low expression in the brain (Kannan \& Fridell 2013). The different Ilp proteins in Drosophila appear to have specialized functions depending on the substrate affected and/or on the metabolic state of the fly. Ilp2 appears to be coupled to trehalose levels (Kannan \& Fridell 2013). Ilp2, Ilp3, and Ilp5 are all secreted in response to nutrient cues (Chatterjee et al. 2014), mirroring mammalian insulin signaling. However, during starvation, Ilp2 is unchanged, whereas Ilp3 and Ilp5 decrease expression (Ikeya et al. 2002). Furthermore, Ilp3 and Ilp5 increase with Ilp2 knockdown (Nassel et al. 2013). In our results, the changes in the expression profile were the same for Ilp2, Ilp3, and Ilp5 (decreased Ilp2 with increased Ilp3

Published by Bioscientifica Ltd 
and Ilp5) as in previous reports where IPCs were ablated (Kannan \& Fridell 2013), but not for adipokinetic hormone $(A k h)$, which functions as glucagon in fly (Kim \& Rulifson 2004, Lee \& Park 2004, Bharucha et al. 2008, Buch et al. 2008, Braco et al. 2012, Matsuda et al. 2015) and increases when IPCs are ablated (Buch et al. 2008). In our experiments, Ilp2 may be decreased in response to a cell-autonomous starvation signal, whereas Ilp3 and Ilp5 are increased in response to decreased Ilp2 expression. And the increased Ilp3 and/or Ilp5 from the IPCs may be responsible for inhibiting $A k h$ does as insulin for glucagon in humans.

Lastly, in a qualitative description of insulin signaling using $t G P H$ transgenic flies, we observed that fat body cells from larva lacking Tmem18 in the IPCs did not respond normally to feeding/insulin. Thus, loss of Tmem18 appears to make cells insulin resistant, which makes sense given that circulating glucose and trehalose were elevated. Moreover, these same high levels of substrate in addition to the augmented Ilp expression are likely responsible for the decrease in $A k h$ expression. Given all the above, it appears that flies lacking Tmem18 are unable to respond to insulin or have impaired insulin secretion, leaving elevated substrate levels in circulation and storage, and disrupting the normal function of the Ilp proteins, InR, and AKH (Fig. 6).

This study is not without limitations. The SNPs we list as likely to be important for metabolism have yet unproven links to TMEM18 regulation/function. Thus, we caution that these findings from the human data should be interpreted as correlative. Although these findings were enough to justify our following series of experiments, the link between these SNPs and TMEM18 requires further validation.

In conclusion, we present a molecular description for the function of TMEM18. We found clues from human SNP data that TMEM18 may be involved in metabolic signaling involving insulin. From experiments with the ortholog of TMEM18 in D. melanogaster, we show that knocking down Tmem18 in cells analogous to human pancreatic beta cells induced changes in substrate levels and insulin signaling, suggesting a metabolic state resembling type 2 diabetes or long-term starvation. From all this, we suggest that TMEM18 is an interesting gene for further studying the etiology or treatment of obesity, metabolic syndrome, and type 2 diabetes.

\section{Supplementary data}

This is linked to the online version of the paper at http://dx.doi.org/10.1530/ JOE-16-0040.

\section{Declaration of interest}

The authors declare that there is no conflict of interest that could be perceived as prejudicing the impartiality of the research reported.

\section{Funding}

This study was supported by the Swedish Research Council and the Åhlens Foundation.

\section{Author contribution statement}

All authors have read and approved this version of the manuscript. Dr Wiemerslage wrote the majority of the manuscript, planned/organized a majority of the experiments, and analyzed a majority of the data. Gohel wrote parts of this manuscript and collected/analyzed results pertaining to the biochemical and molecular experiments as well as some of the behavioral experiments. Maestri performed/analyzed some of the molecular experiments. Hilmarsson collected/analyzed results pertaining to the behavioral experiments. Dr Mickael performed the phylogenetic analysis and contributed to its explanation. Dr Fredriksson was involved in the initial design of the study and provided insights into the phylogenetic analysis. Dr Williams helped with the writing of this manuscript and was involved in the planning/development of many of the experimental procedures. Dr Schiöth helped with the writing of this manuscript, oversaw the project, and was involved in the planning of many of the experimental procedures.

\section{Acknowledgments}

The authors thank the investigators of the GIANT, MAGIC, DIAGRAM, GLGC, and CARDIoGRAM consortia for graciously making their data publicly available, as well as the developers of the Genome Browser, RegulomeDB, mrSNP, Human eFP Browser, FlyAtlas 2, and the Human Protein Browser for also making their online tools free and open to the public.

\section{References}

Almen MS, Jacobsson JA, Shaik JH, Olszewski PK, Cedernaes J, Alsio J, Sreedharan S, Levine AS, Fredriksson R, Marcus C, et al. 2010 The obesity gene, TMEM18, is of ancient origin, found in majority of neuronal cells in all major brain regions and associated with obesity in severely obese children. BMC Medical Genetics 1158.

Altschul SF, Gish W, Miller W, Myers EW \& Lipman DJ 1990 Basic local alignment search tool. Journal of Molecular Biology 215 403-410. (doi:10.1016/S0022-2836(05)80360-2)

Barness LA, Opitz JM \& Gilbert-Barness E 2007 Obesity: genetic, molecular, and environmental aspects. American Journal of Medical Genetics A 143A 3016-3034. (doi:10.1002/ajmg.a.32035)

Bharucha KN, Tarr P \& Zipursky SL 2008 A glucagon-like endocrine pathway in Drosophila modulates both lipid and carbohydrate homeostasis. Journal of Experimental Biology 211 3103-3110. (doi:10.1242/jeb.016451)

Boyle AP, Hong EL, Hariharan M, Cheng Y, Schaub MA, Kasowski M, Karczewski KJ, Park J, Hitz BC, Weng S, et al. 2012 Annotation of functional variation in personal genomes using RegulomeDB. Genome Research 22 1790-1797. (doi:10.1101/gr.137323.112)

Braco JT, Gillespie EL, Alberto GE, Brenman JE \& Johnson EC 2012 Energy-dependent modulation of glucagon-like signaling in Drosophila via the AMP-activated protein kinase. Genetics 192 457-466. (doi:10.1534/genetics.112.143610)

Published by Bioscientifica Ltd. 
Brand AH \& Perrimon N 1993 Targeted gene-expression as a means of altering cell fates and generating dominant phenotypes. Development 118 401-415

Britton JS, Lockwood WK, Li L, Cohen SM \& Edgar BA 2002 Drosophila's insulin/PI3-kinase pathway coordinates cellular metabolism with nutritional conditions. Developmental Cell 2 239-249. (doi:10.1016/ S1534-5807(02)00117-X)

Broughton SJ, Piper MD, Ikeya T, Bass TM, Jacobson J, Driege Y, Martinez P, Hafen E, Withers DJ, Leevers SJ, et al. 2005 Longer lifespan, altered metabolism, and stress resistance in Drosophila from ablation of cells making insulin-like ligands. PNAS 102 3105-3110. (doi:10.1073/pnas.0405775102)

Buch S, Melcher C, Bauer M, Katzenberger J \& Pankratz MJ 2008 Opposing effects of dietary protein and sugar regulate a transcriptional target of Drosophila insulin-like peptide signaling. Cell Metabolism 7 321-332. (doi:10.1016/j.cmet.2008.02.012)

Calle EE, Rodriguez C, Walker-Thurmond K \& Thun MJ 2003 Overweight, obesity, and mortality from cancer in a prospectively studied cohort of U.S. adults. New England Journal of Medicine $\mathbf{3 4 8}$ 1625-1638.

Cao J, Ni J, Ma W, Shiu V, Milla LA, Park S, Spletter ML, Tang S, Zhang J, Wei X, et al. 2014 Insight into insulin secretion from transcriptome and genetic analysis of insulin-producing cells of Drosophila. Genetics 197 175-192. (doi:10.1534/ genetics.113.160663)

Chatterjee D, Katewa SD, Qi Y, Jackson SA, Kapahi P \& Jasper H 2014 Control of metabolic adaptation to fasting by dILP6-induced insulin signaling in Drosophila oenocytes. PNAS 111 17959-17964. (doi:10.1073/pnas.1409241111)

Deveci M, Catalyurek UV \& Toland AE 2014 mrSNP: software to detect SNP effects on microRNA binding. BMC Bioinformatics 1573. (doi:10.1186/1471-2105-15-73)

Dupuis J, Langenberg C, Prokopenko I, Saxena R, Soranzo N, Jackson AU, Wheeler E, Glazer NL, Bouatia-Naji N, Gloyn AL et al. 2010 New genetic loci implicated in fasting glucose homeostasis and their impact on type 2 diabetes risk. Nature Genetics $\mathbf{4 2}$ 105-116.

Haslam DW \& James WP 2005 Obesity. Lancet 366 1197-1209. (doi:10.1016/S0140-6736(05)67483-1)

Ikeya T, Galic M, Belawat P, Nairz K \& Hafen E 2002 Nutrient-dependent expression of insulin-like peptides from neuroendocrine cells in the CNS contributes to growth regulation in Drosophila. Current Biology 12 1293-1300. (doi:10.1016/S0960-9822(02)01043-6)

Ja WW, Carvalho GB, Mak EM, de la Rosa NN, Fang AY, Liong JC, Brummel T \& Benzer S 2007 Prandiology of Drosophila and the CAFE assay. PNAS 104 8253-8256. (doi:10.1073/ pnas.0702726104)

Jurvansuu JM \& Goldman A 2011 Obesity risk gene TMEM18 encodes a sequence-specific DNA-binding protein. PLoS ONE 6 e25317.

Jurvansuu J, Zhao Y, Leung DS, Boulaire J, Yu YH, Ahmed S \& Wang S 2008 Transmembrane protein 18 enhances the tropism of neural stem cells for glioma cells. Cancer Research 68 4614-4622. (doi:10.1158/0008-5472.CAN-07-5291)

Kalnina I, Zaharenko L, Vaivade I, Rovite V, Nikitina-Zake L, Peculis R, Fridmanis D, Geldnere K, Jacobsson JA, Almen MS, et al. 2013 Polymorphisms in FTO and near TMEM18 associate with type 2 diabetes and predispose to younger age at diagnosis of diabetes. Gene 527 462-468. (doi:10.1016/j.gene.2013.06.079)

Kannan K \& Fridell YW 2013 Functional implications of Drosophila insulin-like peptides in metabolism, aging, and dietary restriction. Frontiers in Physiology 4288.

Kim SK \& Rulifson EJ 2004 Conserved mechanisms of glucose sensing and regulation by Drosophila corpora cardiaca cells. Nature $\mathbf{4 3 1}$ 316-320. (doi:10.1038/nature02897)

Lee G \& Park JH 2004 Hemolymph sugar homeostasis and starvationinduced hyperactivity affected by genetic manipulations of the adipokinetic hormone-encoding gene in Drosophila melanogaster. Genetics 167 311-323. (doi:10.1534/genetics.167.1.311)

Manning AK, Hivert MF, Scott RA, Grimsby JL, Bouatia-Naji N, Chen H, Rybin D, Liu CT, Bielak LF, Prokopenko I, et al. 2012 A genome-wide approach accounting for body mass index identifies genetic variants influencing fasting glycemic traits and insulin resistance. Nature Genetics 44 659-669.

Matsuda H, Yamada T, Yoshida M \& Nishimura T 2015 Flies without trehalose. Journal of Biological Chemistry 290 1244-1255. (doi:10.1074/ jbc.M114.619411)

Nassel DR, Kubrak OI, Liu Y, Luo J \& Lushchak OV 2013 Factors that regulate insulin producing cells and their output in Drosophila. Frontiers in Physiology 4252.

Rask-Andersen M, Jacobsson JA, Moschonis G, Chavan RA, Sikder MA, Allzen E, Alsio J, Chrousos GP, Manios Y, Fredriksson R, et al. 2012 Association of TMEM18 variants with BMI and waist circumference in children and correlation of mRNA expression in the PFC with body weight in rats. European Journal of Human Genetics 20 192-197. (doi:10.1038/ ejhg.2011.176)

Robinson SW, Herzyk P, Dow JAT \& Leader DP 2013 FlyAtlas: database of gene expression in the tissues of Drosophila melanogaster. Nucleic Acids Research 41 D744-D750.

Rohde K, Keller M, Klos M, Schleinitz D, Dietrich A, Schon MR, Gartner D, Lohmann T, Dressler M, Stumvoll M, et al. 2014 Adipose tissue depot specific promoter methylation of TMEM18. Journal of Molecular Medicine 92 881-888. (doi:10.1007/s00109014-1154-1)

Rosenbloom KR, Dreszer TR, Long JC, Malladi VS, Sloan CA, Raney BJ, Cline MS, Karolchik D, Barber GP, Clawson H, et al. 2012 ENCODE whole-genome data in the UCSC Genome Browser: update 2012. Nucleic Acids Research 40 D912-D917.

Rosenbloom KR, Sloan CA, Malladi VS, Dreszer TR, Learned K, Kirkup VM, Wong MC, Maddren M, Fang R, Heitner SG, et al. 2013 ENCODE data in the UCSC Genome Browser: year 5 update. Nucleic Acids Research 41 D56-D63.

Rulifson EJ, Kim SK \& Nusse R 2002 Ablation of insulin-producing neurons in flies: growth and diabetic phenotypes. Science 296 1118-1120. (doi:10.1126/science.1070058)

Slack C, Werz C, Wieser D, Alic N, Foley A, Stocker H, Withers DJ, Thornton JM, Hafen E \& Partridge L 2010 Regulation of lifespan, metabolism, and stress responses by the Drosophila SH2B protein, Lnk. PLoS Genetics 6 e1000881.

Soranzo N, Sanna S, Wheeler E, Gieger C, Radke D, Dupuis J, BouatiaNaji N, Langenberg C, Prokopenko I, Stolerman E, et al. 2010 Common variants at 10 genomic loci influence hemoglobin $\mathrm{A}(1)(\mathrm{C})$ levels via glycemic and nonglycemic pathways. Diabetes 59 3229-3239.

Speliotes EK, Willer CJ, Berndt SI, Monda KL, Thorleifsson G, Jackson AU, Lango Allen H, Lindgren CM, Luan J, Magi R, et al. 2010 Association analyses of 249,796 individuals reveal 18 new loci associated with body mass index. Nature Genetics $\mathbf{4 2}$ 937-948.

Tennessen JM, Baker KD, Lam G, Evans J \& Thummel CS 2011 The Drosophila estrogen-related receptor directs a metabolic switch that supports developmental growth. Cell Metabolism 13 139-148. (doi:10.1016/j.cmet.2011.01.005)

Teslovich TM, Musunuru K, Smith AV, Edmondson AC, Stylianou IM, Koseki M, Pirruccello JP, Ripatti S, Chasman DI, Willer CJ, et al. 2010 Biological, clinical and population relevance of 95 loci for blood lipids. Nature 466 707-713.

Thorleifsson G, Walters GB, Gudbjartsson DF, Steinthorsdottir V, Sulem P, Helgadottir A, Styrkarsdottir U, Gretarsdottir S, Thorlacius S, Jonsdottir I, et al. 2009 Genome-wide association yields new sequence variants at seven loci that associate with measures of obesity. Nature Genetics 41 18-24. (doi:10.1038/ng.274) http://joe.endocrinology-journals.org DOI: 10.1530/JOE-16-0040
(C) 2016 Society for Endocrinology Printed in Great Britain 
Uhlen M, Oksvold P, Fagerberg L, Lundberg E, Jonasson K, Forsberg M, Zwahlen M, Kampf C, Wester K, Hober S, et al. 2010 Towards a knowledge-based Human Protein Atlas. Nature Biotechnology 28 1248-1250. (doi:10.1038/nbt1210-1248)

Uhlén M, Fagerberg L, Hallström BM, Lindskog C, Oksvold P, Mardinoglu A, Sivertsson Å, Kampf C, Sjöstedt E, Asplund A, et al. 2015 Tissue-based map of the human proteome. Science $\mathbf{3 4 7}$ 1260419.

Wang S, Tulina N, Carlin DL \& Rulifson EJ 2007 The origin of islet-like cells in Drosophila identifies parallels to the vertebrate endocrine axis. PNAS 104 19873-19878. (doi:10.1073/pnas.0707465104)

Willer CJ, Speliotes EK, Loos RJ, Li S, Lindgren CM, Heid IM, Berndt SI, Elliott AL, Jackson AU, Lamina C, et al. 2009 Six new loci associated with body mass index highlight a neuronal influence on body weight regulation. Nature Genetics $\mathbf{4 1} 25-34$.
Williams MJ, Almen MS, Fredriksson R \& Schioth HB 2012 What model organisms and interactomics can reveal about the genetics of human obesity. Cellular and Molecular Life Sciences 69 3819-3834. (doi:10.1007/s00018-012-1022-5)

Williams MJ, Goergen P, Rajendran J, Klockars A, Kasagiannis A, Fredriksson R \& Schioth HB 2014a Regulation of aggression by obesity-linked genes TfAP-2 and Twz through octopamine signaling in Drosophila. Genetics 196 349-362.

Williams MJ, Wang Y, Klockars A, Monica Lind P, Fredriksson R \& Schioth HB 2014b Exposure to bisphenol A affects lipid metabolism in Drosophila melanogaster. Basic \& Clinical Pharmacology \& Toxicology 114 414-420.

Winter D, Vinegar B, Nahal H, Ammar R, Wilson GV \& Provart NJ 2007 An "Electronic Fluorescent Pictograph" browser for exploring and analyzing large-scale biological data sets. PLOS ONE 2 e718.

Received in final form 21 March 2016

Accepted 29 March 2016

Accepted Preprint published online 30 March 2016
(C) 2016 Society for Endocrinology Printed in Great Britain
Published by Bioscientifica Ltd. 